\title{
Evaluating the sensitivity of a passive diarrheal disease surveillance system during a post-drought
}

\author{
Nhlanhla Nhlabatsi ${ }^{\star 1}$, Vusie Lokotfwako', Phinda Khumalo', Siphiwe M. Shongwe-Gama², \\ Maria Dlamini', Zanela Simelane ${ }^{1}$, Thulani Maphosa ${ }^{2}$ and Harriet Nuwagaba- \\ Biribonwoha $^{2}$
}

${ }^{1}$ Ministry of Health, Mbabane, Swaziland; ${ }^{2}$ CAP Columbia University, Mbabane, Swaziland

\section{Objective}

To evaluate the difference in sensitivity between passive and active diarrheal and malnutrition disease surveillance system post-drought period in Swaziland

\section{Introduction}

Over the past decade Swaziland has experienced recurring drought episodes. In 2016 the country experienced challenges regarding water supplies in both urban and rural areas due to the drought impact. A rapid health and Nutrition Assessment was conducted in 2016 revealed an increase in number of cases of acute watery diarrhea of all age groups. While there is a high demand for epidemiological data in the country a passive system through Health Management Information System (HMIS) and Immediate Disease Notification System (IDNS) has been used to monitor acute watery diarrhea and a set of priority notifiable diseases in the country.

\section{Methods}

An active sentinel surveillance system was set up in four regional hospitals for monitoring of all diarrheal cases of the under-fives. A data abstraction form was developed and used to extract data from outpatient registers and inpatient mainly from the children's ward over a period of 15 weeks. Two surveillance officers trained on Integrated Disease Surveillance and Response (IDSR) collected and analyzed on weekly basis and further compared with data from a passive surveillance system that included the HMIS and IDNS.

\section{Results}

While acute gastroenteritis was the most prevalent type of diarrheal disease (93\%), about $35.5 \%$ (1788 in active surveillance vs 1147 passive surveillance) of the cases of diarrheal cases are being underreported in the passive surveillance. Similar observation was made on malnutrition with more than $51 \%$ of the cases not reported in passive surveillance (186 cases vs 91 cases).

\section{Conclusions}

The process exposed gaps in data collected for passive surveillance and also differing data standards indicating inconsistency and under reporting which may be misleading for public health purposes. Low sensitivity in terms missing cases within the passive surveillance was observed when comparing within the active surveillance sentinel sites. It was also noted that having multiple data sources poses challenges in the country as they provide varying diseases trends and burden estimate.

\section{Keywords}

Sensitivity; Surveillance; Swaziland

\section{Acknowledgments}

1. Ministry of Health, Mbabane, Swaziland

2. ICAP, Columbia University, Mailman School of Public Health, Mbabane, Swaziland
3. Columbia University, Department of Epidemiology, Mailman School of Public Health, New York, United States of America

\section{References}

1. WHO. Integrated Disease Surveillance and Response

*Nhlanhla Nhlabatsi

E-mail:ntini0064@gmail.com 